\title{
Two-Dimensional Surface Magnetism in the Bulk Paramagnetic Intermetallic Alloy CoAl(100)
}

\author{
V. Rose* \\ Center for Nanoscale Materials, Argonne National Laboratory, Argonne, Illinois 60439, USA \\ K. Brüggemann, R. David, and R. Franchy ${ }^{\dagger}$ \\ Institute for Surfaces and Interfaces (ISG-3), Research Center Jülich, D-52425 Jülich, Germany
}

(Received 25 September 2006; published 17 January 2007)

\begin{abstract}
Utilizing a combination of the in situ magneto-optical Kerr effect and scanning tunneling microscopy and spectroscopy measurements, we show that the (100) surface of the $B 2$ bulk paramagnetic CoAl is an excellent representation of a two-dimensional ferromagnet. The order-parameter critical exponent $\beta=$ $0.22 \pm 0.02$ is determined, which is the universal signature of a finite-size two-dimensional $X Y$ behavior. The Curie temperature is found to be $T_{c}=90 \mathrm{~K}$. The magnetism can be explained by the appearance of Co antisite atoms at the surface.
\end{abstract}

Magnetic surfaces continue to attract considerable interest from both academic and technological points of view. This, on the one hand, is stimulated by the exceptional nature of thin magnetic films giving rise to novel magnetic phenomena that have a great impact on the general understanding of magnetism. On the other hand, inevitable technological advances producing new materials drive it, such as in magnetoelectronic devices, allowing examination of new unexplored physical regimes. In particular, in the last few years, much effort was expended discovering new magnetic materials that are nonmagnetic in the bulk but exhibit intrinsic ferromagnetism at the surface [1-3]. A material with a magnetic surface and nonmagnetic bulk is extremely attractive for technical applications since thin films of such a material would provide a natural magnetic multilayer with perfect matching for the electronic potentials. Consequently, the unwanted strong interface conduction electron scattering that normally arises at the nonmagnetic-magnetic interface could be eliminated. Unfortunately, most of the proposed paramagnetic metallic materials with magnetic surface may be very hard to realize since they are usually based on disordered alloys, in which segregation at the surface is expected that, on its part, could largely suppress the surface magnetism [2].

In this Letter we present in situ surface magneto-optical Kerr effect (MOKE), scanning tunneling microscopy (STM) and scanning tunneling spectroscopy (STS) measurements of the (100) surface of the bulk paramagneticordered compound CoAl. A general consensus exists that perfectly ordered $\mathrm{Co}_{50} \mathrm{Al}_{50}$ (at. \%) is not magnetic even at very low temperatures [4-6]. Nevertheless, our investigations demonstrate that bulk paramagnetic CoAl exhibits a ferromagnetic phase at the surface after standard cleaning treatment in ultrahigh vacuum (UHV) that is induced by antisite atom segregation. The $\beta$ phase of CoAl crystallizes in the $B 2$ (CsCl-type) structure in a wide concentration range around the stoichiometric composition. The crystal field quenches the free-ion orbital momentum of $\mathrm{Co}$ and long-range magnetic order is suppressed in the CoAl bulk. In the case of the nonstoichiometric compound, lowtemperature magnetic susceptibility studies show that, for Co content less than 50 at. \%, CoAl is always paramagnetic [7]. Above 57.9 at. \% Co, the bulk becomes ferromagnetic, with the Curie temperature $T_{c}$ increasing to about $120 \mathrm{~K}$ [7]. Nonetheless, even slight deviations from the ideal stoichiometry in the bulk or an extended region below the surface, which are always present in a real crystal, can significantly affect the surface composition due to surface segregation [8]. Four stable surface phases have been determined by density-functional theory (DFT) calculations for the ordered $\mathrm{CoAl}(100)$ compound, namely, the pure $\mathrm{Al}$ termination, phase separation, with the first and third layers consisting only of Co antisite $\left(\mathrm{Co}_{\mathrm{AS}}\right)$ atoms and two stable superstructures, in which $\mathrm{Al}$ and $\mathrm{Co}_{\mathrm{AS}}$ atoms exhibit chemical order in the top layer [9]. Previous electron energy loss spectroscopy investigations of clean $\mathrm{CoAl}(100)$ have shown that phase separation can be ruled out [10]. In addition, more recent Auger electron spectroscopy (AES) measurements with both an electron gun in normal incidence geometry and primary electrons under grazing incidence conditions support the idea that the $\mathrm{Co} / \mathrm{Al}$ ratio varies within the top atomic layers [11]. Thus, the combination of calculations and experiments suggests that the topmost surface layer of $\operatorname{CoAl}(100)$ consists of both $\mathrm{Al}$ and $\mathrm{Co}_{\mathrm{AS}}$ atoms. In the surface slab, $\mathrm{Co}_{\mathrm{AS}}$ and Co (on regular lattice sites) atoms then may form clusters that exhibit an effective magnetic moment, e.g., with $5.9 \mu_{\mathrm{B}}$ for clusters consisting of 9 Co atoms [4].

The experiments reported were performed in two separate ultrahigh vacuum (UHV) systems with base pressures better than $1 \times 10^{-10}$ mbar. The first was equipped with facilities for in situ surface magneto-optical Kerr effect (MOKE), a cylindrical mirror analyzer for AES allowing surface chemical control, and a three-grid optic for lowenergy electron diffraction (LEED). The MOKE experiments were recorded in longitudinal geometry. A laser 
diode with a wavelength of $670 \mathrm{~nm}$ was used as a light source for the MOKE measurements, and Kerr ellipticity was measured to obtain magnetization curves. Laser and detector were outside the UHV chamber and a $\lambda / 4$ plate was used to compensate for the effect of the windows on the linear polarization of the light. The sample holder was attached to a liquid-He cryostat. Sample temperature was measured by a WRe $25 \%$-WRe $3 \%$ thermocouple mounted directly to the back of the sample. The second UHV chamber was used to perform scanning tunneling microscopy (STM) and scanning tunneling spectroscopy (STS) measurements. It was also equipped with AES and LEED. The STM images were recorded at room temperature in the constant-current mode. The STS spectra were measured at $50 \mathrm{~K}$. Current-voltage $I(U)$ characteristics were taken by stabilizing the tip over the corresponding part of the surface with a bias voltage of $-2.0 \mathrm{~V}$ and a tunneling current of $0.43 \mathrm{nA}$. Then, a voltage range from -2 to $2 \mathrm{~V}$ was applied, while the distance between the tip and sample was kept constant.

The CoAl(100) single crystal had a diameter of $10 \mathrm{~mm}$ and a thickness of $2.1 \mathrm{~mm}$. The accuracy of the orientation, controlled by $x$-ray diffraction, was $0.1^{\circ}$ after mechanical polishing. A clean $\mathrm{CoAl}(100)$ surface was obtained after treating the sample by repeated cycles of $\mathrm{Ar}^{+}$ion sputtering at $1 \mathrm{kV}$ for $20 \mathrm{~min}\left(\mathrm{Ar}^{+}\right.$pressure of $5 \times 10^{-6} \mathrm{mbar}$, sample current of $2 \mu \mathrm{A}$ ) and subsequent annealing under UHV at $1470 \mathrm{~K}(5 \mathrm{~min})$ by electron bombardment. The clean $\mathrm{CoAl}(100)$ surface shows a $(1 \times 1)$ LEED pattern with sharp Bragg reflections, high contrast, and very low background. The AES spectrum exhibits only the characteristic transitions of Co and Al. Figure 1 shows a STM

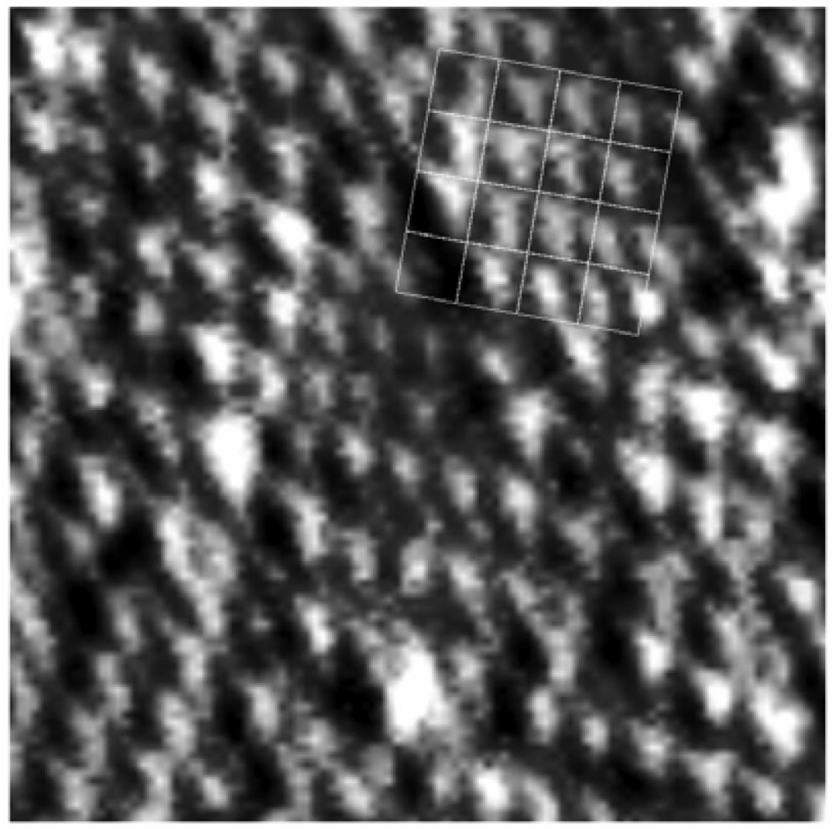

FIG. 1. STM image $\left(4 \times 4 \mathrm{~nm}^{2}\right)$ of $\mathrm{CoAl}(100)$. A grid of unit cells is marked for illustration. $U_{t}=-1.5 \mathrm{~V}, I_{t}=1 \mathrm{nA}$. image $\left(4 \times 4 \mathrm{~nm}^{2}\right)$ of the clean $\operatorname{CoAl}(100)$ surface with atomic resolution. Some rectangular unit cells with a lattice constant of $a=0.28 \mathrm{~nm}$ are marked for illustration.

After standard cleaning treatment, the $\operatorname{CoAl}(100)$ surface exhibits a ferromagnetic-paramagnetic phase transition at low temperatures. In Fig. 2(a), we present a measurement of the Kerr ellipticity $\varepsilon$ versus temperature $T$. The external magnetic field $H$ is applied parallel to the crystallographic [100] direction of the CoAl(100) surface. For $T>90 \mathrm{~K}$, the hysteresis loops collapse and consequently yield $\varepsilon=0$. Below $90 \mathrm{~K}$, the Kerr ellipticity $\varepsilon$ increases steadily for decreasing $T$. The inset in Fig. 2(a) exemplifies two magnetization reversal loops taken at 76 and $91 \mathrm{~K}$, respectively. For $T<90 \mathrm{~K}$, all measured hysteresis loops exhibit a squarelike shape, such as for the case of $76 \mathrm{~K}$. Although no direct information can be obtained regarding the absolute magnitude of magnetization, the angle of Kerr rotation is proportional to $M$ in terms of molecular-orbital energy levels [12]. For temperatures very close to the Curie point $T_{c}$, critical fluctuations result in a power law dependence $M(T) \propto \varepsilon(T) \propto\left(1-T / T_{c}\right)^{\beta}$ with a critical exponent $\beta$. To identify the universality class of the clean $\mathrm{CoAl}(100)$ surface, the evolution of the spontaneous magnetization $M$ with temperature $T$ was compared to a power law $\left(1-T / T_{c}\right)^{\beta}$ by plotting $\log (\varepsilon)$ versus $\log (1-$ $T / T_{c}$ ) in Fig. 2(b). The data are fitted by a straight line, yielding $T_{c}=90 \mathrm{~K}$, and a critical exponent of $\beta=0.22 \pm$ 0.02 . This value is different from the one expected for the 2D-Ising universality class $(\beta=0.125)$ but rather close to the universal signature of a finite-size $2 \mathrm{D}-X Y$ behavior ( $\left.\beta=3 \pi^{2} / 128 \approx 0.23\right)$ [13]. Thus, the (100) surface of bulk paramagnetic $\mathrm{CoAl}$ is an excellent representation of a 2D ferromagnet. Theoretically, 2D ferromagnetism should not occur for isotropic nearest-neighbor exchange, except in the Ising model. Thus, the existence of surface ferromagnetism in $\mathrm{CoAl}$ proves that the interactions are generally not isotropic and that anisotropies play a crucial role. For the sake of completeness, we should mention that a transition temperature of $T_{c}=90 \mathrm{~K}$ would correspond to an extensive off-stoichiometry in the 3D bulk of $\mathrm{Co}_{x} \mathrm{Al}_{100-x}$ with $x \sim 57$ [14], which can be clearly ruled out for our crystal. The coercivity $H_{c}$ as a function of $T$ is shown in Fig. 2(c). The dashed line is a guide to the eye and represents the power law fit derived from the temperature dependence of $\varepsilon$. The coercive field $H_{c}$ ranges from $2.8 \mathrm{Oe}$ at $76 \mathrm{~K}$ to only $0.3 \mathrm{Oe}$ at $89 \mathrm{~K}$ and is zero for $T>$ $90 \mathrm{~K}$. Qualitatively, the ellipticity $\varepsilon$ exhibits the same temperature dependence as $H_{c}$. While for the equally prepared clean $\mathrm{CoAl}(100)$ surface both the Curie temperature and critical exponent are highly reproducible, variations in the standard cleaning treatment may influence these parameters.

Surface magnetism in bulk paramagnetic $\mathrm{CoAl}$ involves the availability of permanent magnetic moments at the surface. These can be induced by the discussed surface 


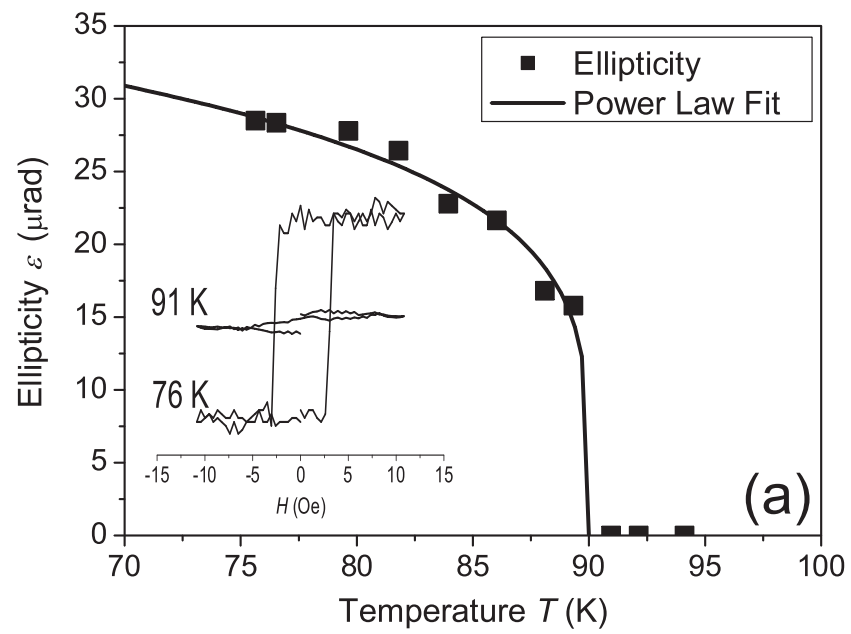

segregation of $\mathrm{Co}_{\mathrm{AS}}$ atoms giving rise to a mixture of $\mathrm{Al}$ and $\mathrm{Co}_{\mathrm{AS}}$ atoms at the surface [8,9,11]. Consequently, $\mathrm{Co}_{\mathrm{AS}}$ atoms together with Co atoms on regular lattice sites form clusters with an effective magnetic moment. A detailed investigation of the mechanism of segregation and aggregation of $\mathrm{Co}_{\mathrm{AS}}$ atoms is extremely difficult. Nevertheless, such data can be obtained by exploiting surface-sensitive spectroscopy techniques. Current-voltage $I(U)$ characteristics recorded equally distributed across the surface allow one to get an insight into the surface termination of CoAl(100). In Fig. 3(a), obviously two different $I(U)$ characteristics can be distinguished that arise due to the dissimilar electronic structures at the surface. The spectra in Fig. 3(a) are calculated from the average of
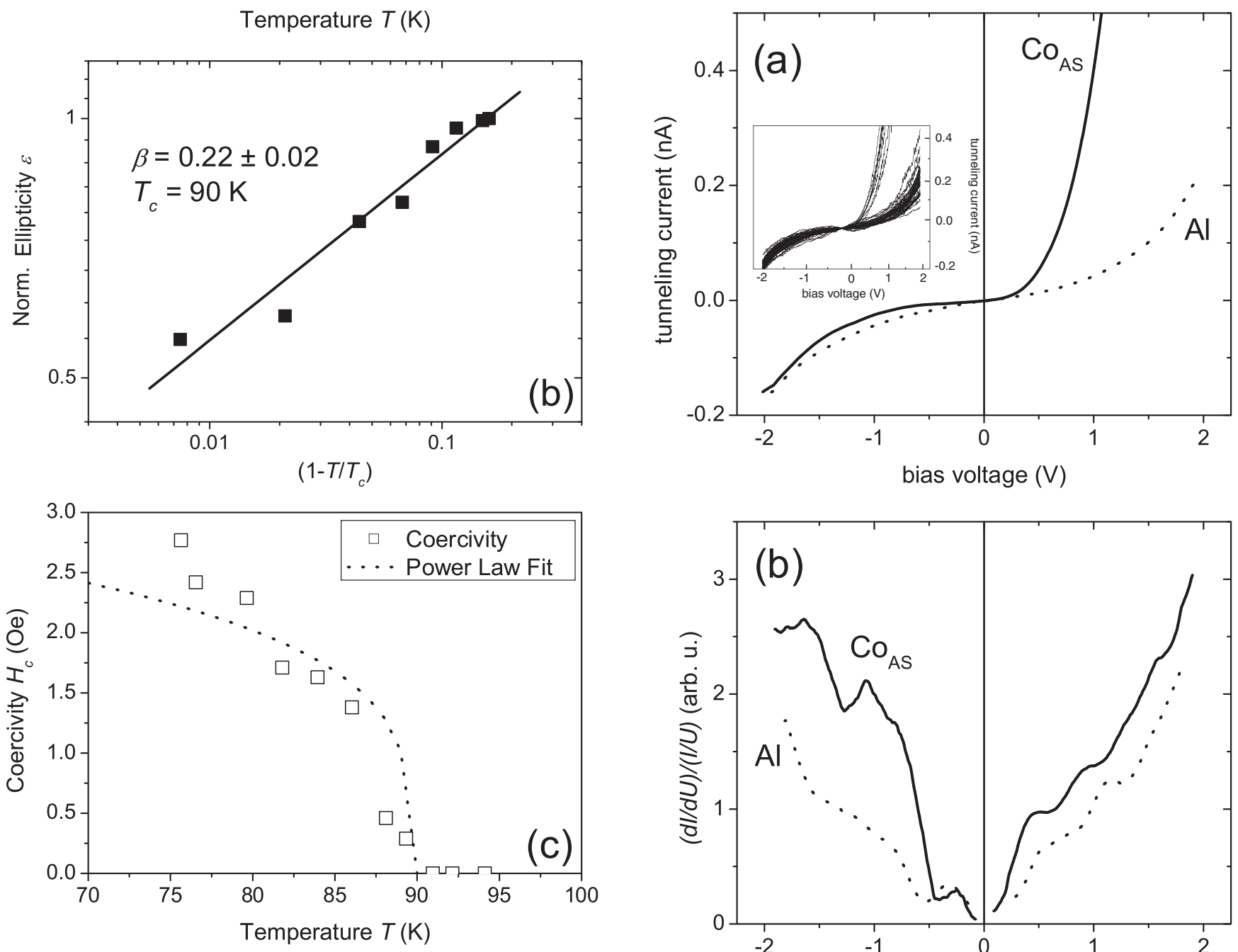

FIG. 2. (a) Kerr ellipticity $\varepsilon$ of $\operatorname{CoAl}(100)$ close to the Curie temperature $T_{c}$. The solid line represents a phenomenological power law fit with critical exponent $\beta=0.22$ and $T_{c}=90 \mathrm{~K}$. The small inset exemplifies two reversal loops taken at 76 and 91 K. (b) Normalized Kerr ellipticity vs reduced temperature, plotted double logarithmically, in order to determine $\beta$. (c) Coercivity $H_{c}$ close to $T_{c}$ with the above fit, provided as a guide to the eyes. $(H||[100])$.

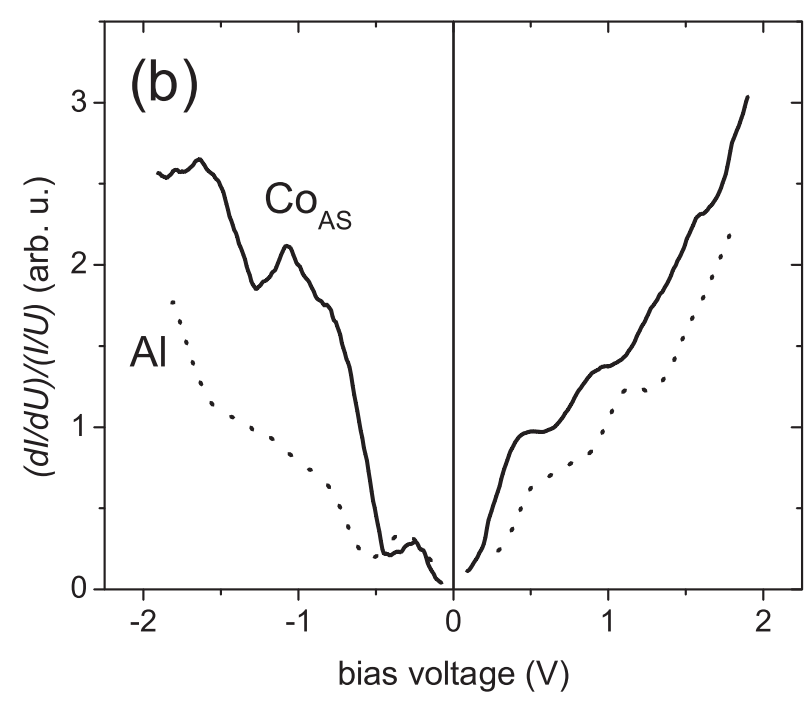

FIG. 3. (a) The two characteristic $I(U)$ curves of the clean $\mathrm{CoAl}(100)$ surface calculated from the average of single $I(U)$ curves (inset) that have been acquired using STS. Spectra have been taken by stabilizing the tip at $U=-2.0 \mathrm{~V}$ and $I=$ $0.43 \mathrm{nA}$. (b) Normalized conductivity $(d I / d U) /(I / U)$ data obtained from the averaged spectra. 
corresponding single $I(U)$ curves [inset in Fig. 3(a)]. The appearance of two different characteristics can be assigned to $\mathrm{Al}$ and $\mathrm{Co}_{\mathrm{AS}}$ atoms in the surface plane, as described in the following. The statistical evaluation shows that around $80 \%$ of the single spectra are of type $\mathrm{Al}$, while $20 \pm 6 \%$ are attributed to $\mathrm{Co}_{\mathrm{AS}}$. In the two proposed ordered surface phases of $\operatorname{CoAl}(100)$ [9], either $1 / 2\left[c(2 \times 2)^{+3 L}\right]$ or $1 / 3$ $[(4 \times 4) \operatorname{diag}]$ of the surface atoms are expected to be $\mathrm{Co}_{\mathrm{AS}}$. However, in our LEED investigations no superstructure is observed indicating that the $\mathrm{Co}_{\mathrm{AS}}$ atoms have no longrange order. For negative bias (corresponding to tunneling from occupied states of the sample into the tip), the general slope of the individual curves is almost the same. Nevertheless, in the averaged spectrum, the decrease of $I$ with decreasing $U$ is more pronounced over Al. For positive voltage (tunneling from the tip into empty states of the sample), the differences in the $I(U)$ characteristics become more obvious. Particularly striking is the extremely strong increase of $I$ above $0.5 \mathrm{~V}$ over $\mathrm{Co}_{\mathrm{AS}}$. In Fig. 3(b), the normalized tunneling conductivity $(d I / d U) /(I / U)$ derived from the $I(U)$ data in Fig. 3(a) is presented. Peaks in the normalized tunneling conductance are related to maxima in the local density of states (LDOS) [15]. For positive bias, both curves exhibit maxima at around 0.5 and $1 \mathrm{~V}$. In addition, for one curve a pronounced maximum is visible at $-1.2 \mathrm{~V}$ accompanied by a peak at $-0.3 \mathrm{~V}$ that are the fingerprint of the occupied majority states in the LDOS of spin polarized $\mathrm{Co}_{\mathrm{AS}}$ atoms [16]. Consequently, the second curve may be assigned to $\mathrm{Al}$ atoms. Nevertheless, more comprehensive calculations of the electronic and magnetic structure would be desirable.

In summary, our results prove the existence of a ferromagnetic surface of the intermetallic alloy $\operatorname{CoAl}(100)$, which otherwise is nonmagnetic in the bulk. The magnetism of this surface is connected with the accumulation of $\mathrm{Co}_{\mathrm{AS}}$ atoms, which (together with Co atoms on regular lattice sites) form clusters that exhibit an effective magnetic moment. The Curie temperature $T_{c}$ amounts to $90 \mathrm{~K}$ after a standard cleaning treatment in UHV. Around $1 / 5$ of the surface sites are found to be occupied by $\mathrm{Co}_{\mathrm{AS}}$ atoms, which is close to the ratio expected for the $(4 \times 4)$ diag phase proposed by earlier DFT calculations [9]. The exceptional properties of $\mathrm{CoAl}(100)$ may provide the opportunity to study itinerant magnetism localized in two dimensions and open up a path for the exploration of novel materials for innovative technical applications.

The project has been completed at Research Center Jülich with the kind support of Professor Harald Ibach. We acknowledge the late René Franchy (see Ref. [17]) for the contributions to the research for this Letter.

*Corresponding author.

Electronic address: vrose@anl.gov.

${ }^{\dagger}$ Deceased.

[1] I. Turek, S. Blügel, and J. Kudrnovsky, Phys. Rev. B 57, R11065 (1998).

[2] A. V. Ponomareva, L. V. Pourovskii, E. I. Isaev, Yu. Kh. Vekilov, B. Johansson, and I. A. Abrikosov, J. Magn. Magn. Mater. 258-259, 128 (2003).

[3] S. Khmelevskyi, P. Mohn, J. Redinger, and M. Weinert, Phys. Rev. Lett. 94, 146403 (2005).

[4] D. J. Sellmeyer, G. R. Caskey, and J. Franz, J. Phys. Chem. Solids 33, 561 (1972).

[5] A. Amamou and F. Gautier, J. Phys. F 4, 563 (1974).

[6] N. I. Kulikov, A. V. Postnikov, G. Borstel, and J. Braun, Phys. Rev. B 59, 6824 (1999).

[7] E. Wachtel, V. Linse, and V. Gerold, J. Phys. Chem. Solids 34, 1461 (1973).

[8] V. Blum, L. Hammer, Ch. Schmidt, W. Meier, O. Wieckhorst, S. Müller, and K. Heinz, Phys. Rev. Lett. 89, 266102 (2002).

[9] O. Wieckhorst, S. Müller, L. Hammer, and K. Heinz, Phys. Rev. Lett. 92, 195503 (2004).

[10] V. Rose, V. Podgursky, I. Costina, and R. Franchy, Surf. Sci. 541, 128 (2003).

[11] V. Rose, V. Podgursky, I. Costina, R. Franchy, and H. Ibach, Surf. Sci. 577, 139 (2005).

[12] M. T. Johnson, P. J. H. Bloemen, F. J. A. den Broeder, and J. J. de Vries, Rep. Prog. Phys. 59, 1409 (1996).

[13] S. T. Bramwell and P. C. W. Holdsworth, J. Phys. Condens. Matter 5, L53 (1993).

[14] J. G. Booth, in Condensed Matter, Landolt-Börnstein, New Series, Group III, Vol. 32B, Pt. 2 (Springer, Berlin/ Heidelberg, 1999), p. 245.

[15] D. A. Bonnell, Scanning Probe Microscopy and Spectroscopy: Theory, Techniques, and Applications (Wiley-VCH, New York, 2001).

[16] N. Stefanou, R. Zeller, and P. H. Dederichs, Phys. Rev. B 35, 2705 (1987).

[17] H. Ibach, T. Raman, A. Otto, L. Baker, and B. Frederick, Surf. Sci. 587, XV (2005). 